\title{
DIFFERENTIAL SPACE-TIME SPREADING USING ITERATIVELY DETECTED SPHERE PACKING MODULATION AND TWO TRANSMIT ANTENNAS
}

\author{
M. El-Hajjar, O. Alamri and L. Hanzo* \\ School of ECS, University of Southampton, SO17 1BJ, UK. \\ Email: 1h@ecs.soton.ac.uk \\ http://www-mobile.ecs.soton.ac.uk
}

\begin{abstract}
A novel Differentially encoded Space-Time Spreading (DSTS) scheme using two transmit antennas and Sphere Packing (SP) is proposed, which we refer to as the DSTS-SP arrangement. The advocated SP-aided system outperforms DSTS dispensing with SP and requires no channel knowledge. We also demonstrate that the performance of DSTS-SP systems can be further improved by serially concatenated convolutional coding and by performing SP-symbol-to-bit demapping as well as channel decoding iteratively. Explicitly, the proposed turbo-detected DSTS-SP scheme exhibits an $E_{b} / N_{0}$ gain of $17.8 d B$ at a Bit Error Rate $(B E R)$ of $10^{-5}$ over an uncoded identical-throughput system and an $E_{b} / N_{0}$ gain of $1.9 \mathrm{~dB}$ over the equivalent $2 \mathrm{bits} / \mathrm{symbol}$ effective throughput QPSK-modulated turbo-detected DSTS scheme.
\end{abstract}

\section{INTRODUCTION}

The detrimental effects of channel fading may be significantly reduced by employing space-time coding aided transmit diversity invoking multiple antennas [1]. Alamouti [2] discovered a witty transmit diversity scheme employing two transmit antennas. The attractive benefits of Alamouti's design motivated Tarokh et al. $[3,4]$ to generalise Alamouti's transmit diversity scheme to an arbitrary number of transmit antennas. In the ensuing era the design of meritorious space-time modulation schemes has attracted considerable research attention $[5,6]$. Inspired by the philosophy of Space-Time Block Codes (STBC), Hochwald et al. [7] proposed the transmit diversity concept known as Space-Time Spreading (STS) for the downlink of Wideband Code Division Multiple Access (WCDMA) that is capable of achieving the highest possible transmit diversity gain. As a further advance, the concept of combining orthogonal transmit diversity designs with the principle of sphere packing modulation was introduced by $\mathrm{Su}$ et al. in [8], where it was demonstrated that the proposed Sphere Packing (SP) aided STBC system was capable of outperforming the conventional orthogonal design based STBC schemes of [2,3].

A common feature of all the above-mentioned schemes is that they use coherent detection, which requires channel knowledge at the receiver. In practice, the channel state information (CSI) of each link between each transmit and each receive antenna pair has to be estimated at the receiver either blindly or using training symbols. However, channel estimation increases both the cost and complexity of the receiver. Furthermore, when the CSI fluctuates dramatically from burst to burst, an increased number training symbols has to be transmitted, potentially resulting in an undesirably high transmission overhead and wastage of transmission power. Therefore, it is beneficial to develop low-complexity techniques that do not require any channel information. In this contri-

* The financial support of Vodafone under the auspices of the Dorothy Hodgkin Postgraduate Award and that of the Ministry of Higher Education of Saudi Arabia, as well as the support of the European Union under the auspices of the Newcom and Phoenix projects is gratefully acknowledged.
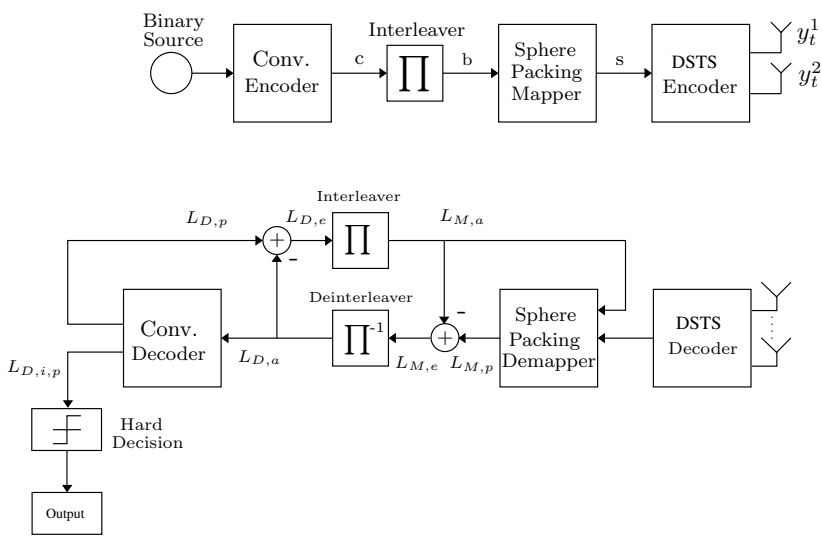

Figure 1: The Turbo Detection Aided DSTS-SP System.

bution, we develop a Differential Space-Time Spreading (DSTS) scheme that requires no CSI. Naturally, this is achieved at the cost of a $3 d B$ performance loss in comparison to the coherent receiver. The proposed DSTS scheme may be readily combined with PSK and SP modulation schemes. Moreover, with the advent of multiple receive antennas, further receive diversity gains can be achieved in addition to the attainable transmit diversity gain.

Iterative decoding of spectrally efficient modulation schemes was considered by several authors [1,9]. In [10], the employment of the turbo principle was considered for iterative soft demapping in the context of multilevel modulation schemes combined with channel decoding, where a soft symbol-to-bit demapper was used between the multilevel demodulator and the binary channel decoder.

Motivated by the performance improvements reported in [8] and [10], the novel DSTS scheme exploits the combined advantages of the differential encoding, the multi-user supporting capability of the STS, as well as the benefits of SP modulation and those of iterative symbol-to-bit demapping and decoding. The DSTS-SP demapper was specifically designed for the sake of accepting the a priori information passed to it from the binary channel decoder as extrinsic information. As a benefit of the proposed solution, it will be demonstrated in Section 6 that the turbo detection aided DSTS$S P$ scheme is capable of providing an $E_{b} / N_{0}$ gain of $17.8 \mathrm{~dB}$ at a Bit Error Rate (BER) of $10^{-5}$.

This paper is organised as follows. In Section 2, a brief system overview is presented, followed by a brief description of the STS design using sphere packing modulation in Section 3. In Section 4, the DSTS encoding and decoding algorithms are highlighted. Section 5 demonstrates how the DSTS-SP demapper is modified for the sake of exploiting the a priori knowledge provided by the channel decoder, while our simulation results and discussions are provided in Section 6. Finally, we conclude in Section 7. 


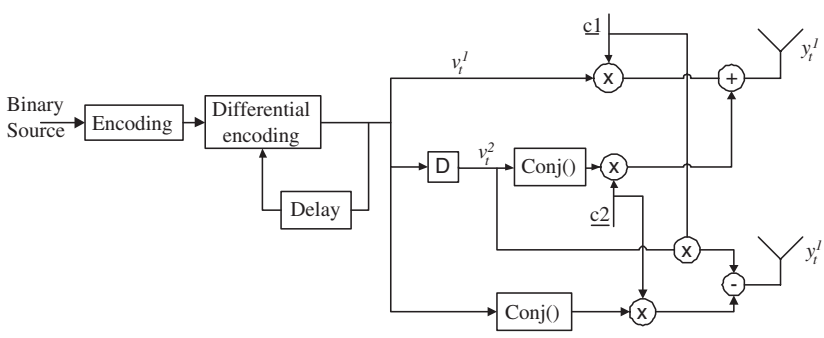

Figure 2: DSTS Encoder Block of Figure 1.

\section{SYSTEM OVERVIEW}

The schematic of the entire system is shown in Figures 1 and 2, where the transmitted source bits are convolutionally encoded and then interleaved by a random bit interleaver. A rate $R=\frac{1}{2}$ recursive systematic convolutional code was employed. After channel interleaving, the DSTS-SP modulator first maps $B$ number of channel-coded bits $\mathbf{b}=b_{0, \ldots, B-1} \in\{0,1\}$ to a sphere packing symbol $s \in S$ such that we have $s=\operatorname{map}_{s p}(\mathbf{b})$, where $B=\log _{2} L$, as outlined in [11]. Subsequently, we have a set of SP symbols that can be transmitted using DSTS within the same time slot using two transmit antennas.

In this treatise, we considered transmission over a correlated narrowband Rayleigh fading channel, associated with a normalised Doppler frequency of $f_{D}=0.01$. The complex Additive White Gaussian Noise (AWGN) of $n=n_{I}+j n_{Q}$ is also added to the received signal, where $n_{I}$ and $n_{Q}$ are two independent zero mean Gaussian random variables having a variance of $\sigma_{n}^{2}=\sigma_{n_{I}}^{2}=$ $\sigma_{n_{Q}}^{2}=N_{0} / 2$ per dimension, with $N_{0} / 2$ representing the doublesided noise power spectral density expressed in $\mathrm{W} / \mathrm{Hz}$.

As shown in Figure 1, the received complex-valued symbols are demapped to their Log-Likelihood Ratio (LLR) representation for each of the $B$ coded bits per DSTS-SP symbol. The $a$ priori LLR values of the demodulator are subtracted from the $a$ posteriori LLR values for the sake of generating the extrinsic LLR values $L_{M, e}$, and then the LLRs $L_{M, e}$ are deinterleaved by a soft-bit deinterleaver, as seen in Figure 1. Next, the soft bits $L_{D, a}$ are passed to the convolutional decoder in order to compute the a posteriori $\mathrm{LLR}$ values $L_{D, p}$ provided by the Max-Log MAP algorithm [12] for all the channel-coded bits. During the last iteration, only the LLR values $L_{D, i, p}$ of the original uncoded systematic information bits are required, which are passed to a hard decision decoder in order to determine the estimated transmitted source bits. The extrinsic information $L_{D, e}$, is generated by subtracting the a priori information from the a posteriori information according to $L_{D, p}-L_{D, a}$, which is then fed back to the DSTS-SP demapper as the a priori information $L_{M, a}$ after appropriately reordering them using the interleaver of Figure 1. The DSTS-SP demapper exploits the a priori information for the sake of providing improved a posteriori LLR values, which are then passed to the channel decoder and then back to the demodulator for further iterations.

\section{STS DESIGN WITH SPHERE PACKING MODULATION}

Alamouti's [2] STBC scheme is described by:

$$
G_{2}\left(x_{1}, x_{2}\right)=\left[\begin{array}{cc}
x_{1} & x_{2} \\
-x_{2}^{*} & x_{1}^{*}
\end{array}\right],
$$

where the rows and columns of Equation (1) represent the temporal and spatial dimensions, corresponding to two consecutive time slots and two transmit antennas, respectively.
It was shown in [8] that the so-called diversity product quantifying the achievable coding advantage ${ }^{1}$ of an orthogonal transmit diversity scheme is determined by the minimum Euclidean distance of the transmitted signal vectors $\left(x_{1}, x_{2}\right)$. Hence, in order to maximise the achievable coding advantage, it was proposed in [8] to use sphere packing schemes that have the best known minimum Euclidean distance in the $2(k+1)$-dimensional real-valued Euclidean space $R^{2(k+1)}$ [13].

It was shown in [7] that the STS-decoded signals represent scaled versions of $x_{1}(t)$ and $x_{2}(t)$ corrupted by the complex-valued AWGN similar to the $G_{2}$ space-time block code of $[2,3]$. This observation implies that the diversity product of STS systems [7] is determined by the minimum Euclidean distance of all legitimate vectors $\left(x_{1}, x_{2}\right)$, where the time index is removed for notational simplicity. According to [7], $x_{1}$ and $x_{2}$ represent conventional BPSK modulated symbols transmitted from the $1^{\text {st }}$ and $2^{\text {nd }}$ antenna and no effort is made to jointly design a signal constellation for the various combinations of $x_{1}$ and $x_{2}$. For the sake of generalising our treatment, let us assume that there are $L$ legitimate vectors $\left(x_{l, 1}, x_{l, 2}\right), l=0,1, \ldots, L-1$, where $L$ represents the number of sphere-packed modulated symbols. The transmitter, then, has to choose the modulated signal from these $L$ legitimate symbols, which have to be transmitted over the two antennas, where the throughput of the system is given by $\left(\log _{2} L\right)$ bits per channel use. In contrast to the independent transmitted signal design of [7], our aim is to design $x_{l, 1}$ and $x_{l, 2}$ jointly, such that they have the best minimum Euclidean distance from all other $(L-1)$ legitimate SP symbols, since this minimises the system's SP symbol error probability. Let $\left(a_{l, 1}, a_{l, 2}, a_{l, 3}, a_{l, 4}\right), l=0,1, \ldots, L-1$, be legitimate phasor points of the four-dimensional real-valued Euclidean space $\mathrm{R}^{4}$, where each of the four elements $a_{l, 1}, a_{l, 2}, a_{l, 3}, a_{l, 4}$ gives one coordinate of the two time-slots' complex-valued phasor points. Hence, $x_{l, 1}$ and $x_{l, 2}$ may be written as

$$
\begin{aligned}
\left\{x_{l, 1}, x_{l, 2}\right\} & =T\left(a_{l, 1}, a_{l, 2}, a_{l, 3}, a_{l, 4}\right) \\
& =\left\{a_{l, 1}+j a_{l, 2}, a_{l, 3}+j a_{l, 4}\right\} .
\end{aligned}
$$

In the four-dimensional real-valued Euclidean space $\mathrm{R}^{4}$, the lattice $D_{4}$ is defined as a sphere packing having the best minimum Euclidean distance from all other $(L-1)$ legitimate constellation points in $\mathrm{R}^{4}$ [13]. More specifically, $D_{4}$ may be defined as a lattice that consists of all legitimate sphere-packed constellation points having integer coordinates $\left[\begin{array}{llll}a_{1} & a_{2} & a_{3} & a_{4}\end{array}\right]$ uniquely and unambiguously describing the legitimate combinations of the modulated symbols $x_{l, 1}$ and $x_{l, 2}$, but subjected to the sphere packing constraint of $a_{1}+a_{2}+a_{3}+a_{4}=k$, where $k$ is an even integer. Assuming that $S=\left\{s^{l}=\left[a_{l, 1}, a_{l, 2}, a_{l, 3}, a_{l, 4}\right] \in \mathrm{R}^{4}\right.$ : $0 \leq l \leq L-1\}$ constitutes a set of $L$ legitimate constellation points from the lattice $D_{4}$ having a total energy of $E \triangleq$ $\sum_{l=0}^{L-1}\left(\left|a_{l, 1}\right|^{2}+\left|a_{l, 2}\right|^{2}+\left|a_{l, 3}\right|^{2}+\left|a_{l, 4}\right|^{2}\right)$, and upon introducing the notation

$$
C_{l}=\sqrt{\frac{2 L}{E}}\left(x_{l, 1}, x_{l, 2}\right), \quad l=0,1, \ldots, L-1,
$$

we have a set of complex constellation symbols, $\left\{C_{l}: 0 \leq l \leq\right.$ $L-1\}$, whose diversity product is determined by the minimum Euclidean distance of the set of $L$ legitimate constellation points in $\mathrm{S}$.

\section{DIFFERENTIAL STS USING TWO TRANSMIT ANTENNAS}

As widely recognised, coherent detection schemes require CSI, which is acquired by transmitting training symbols. However,

\footnotetext{
${ }^{1}$ The diversity product or coding advantage was defined as the estimated gain over an uncoded system having the same diversity order as the coded system [8].
} 
high-accuracy channel estimation imposes a high complexity on the receiver. This renders differential encoding and detection an attractive technique.

In this paper, we assume that no CSI is available at all. The transmitted and received symbols are encoded and decoded based on the differential relationship among subsequent symbols, as in Chapter 11 of [15]. For the sake of simplicity, the power normalisation factor is ignored in our discourse and we consider having a single receive antenna, although these techniques may be generalised for an arbitrary number of receive antennas.

At time instant $t=0$, the arbitrary dummy reference symbols $v_{0}^{1}$ and $v_{0}^{2}$ are transmitted from antennas one and two, respectively, which are known at the receiver. At time instants $t \geq 1$, a block of $2 B$ bits arrive at the encoder, as depicted in Figure 2. Assume that $v_{t}^{1}$ and $v_{t}^{2}$ are the symbols transmitted from antennas one and two, respectively, as seen in Figure 2. Then, $v_{t}^{1}$ and $v_{t}^{2}$ can be differentially encoded as follows:

$$
\begin{aligned}
v_{t}^{1} & =\frac{\left(x_{1} \times v_{t-1}^{1}+x_{2} \times v_{t-1}^{2 *}\right)}{\sqrt{\left(\left|v_{t-1}^{1}\right|^{2}+\left|v_{t-1}^{2}\right|^{2}\right)}} \\
v_{t}^{2} & =\frac{\left(x_{1} \times v_{t-1}^{2}-x_{2} \times v_{t-1}^{1 *}\right)}{\sqrt{\left(\left|v_{t-1}^{1}\right|^{2}+\left|v_{t-1}^{2}\right|^{2}\right)}} .
\end{aligned}
$$

According to Figure 2, the differentially encoded symbols are then spread with the aid of the spreading codes $c_{1}$ and $c_{2}$ to both transmit antennas, where $\underline{c_{1}}$ and $\underline{c_{2}}$ are generated from the same user-specific spreading code $\underline{c}$ by ensuring that the two spreading codes $\underline{c_{1}}$ and $\underline{c_{2}}$ become orthogonal using the simple codeconcatenation rule of:

$$
\left.\begin{array}{rl}
\frac{c_{1}^{T}}{c_{2}^{T}} & =\left[\begin{array}{ll}
\underline{c} & \underline{c}
\end{array}\right] \\
\underline{c} & -\underline{c}
\end{array}\right],
$$

where $T$ denotes the transpose of the vector.

The differentially encoded data is then divided into two substreams as seen in Figure 2 and the two consecutive symbols are then spread to both transmit antennas using the mapping of:

$$
\begin{gathered}
y_{t}^{1}=\underline{c_{1}} \times v_{t}^{1}+\underline{c_{2}} \times v_{t}^{2 *} \\
y_{t}^{2}=\underline{c_{1}} \times v_{t}^{2}-\underline{c_{2}} \times v_{t}^{1 *} .
\end{gathered}
$$

Assuming the channel to be non-dispersive, the received signal at the output of the single receiver antenna can be represented as:

$$
r_{t}=h_{1} \times y_{t}^{1}+h_{2} \times y_{t}^{2}+n_{t},
$$

where $h_{1}$ and $h_{2}$ denote the non-dispersive complex-valued channel impulse responses corresponding to the first and second transmit antennas, respectively, while $n_{t}$ represents the AWGN having a variance of $\sigma_{n}^{2}$.

The received signal $r_{t}$ is then correlated with $\underline{c_{1}}$ and $\underline{c_{2}}$ according to the following operations:

$$
\begin{aligned}
& d_{t}^{1}=\underline{c_{1}^{H}} \times r_{t}=h_{1} \times v_{t}^{1}+h_{2} \times v_{t}^{2}+\underline{c_{1}^{H}} \times n_{t} \\
& d_{t}^{2}=\underline{c_{2}^{H}} \times r_{t}=h_{1} \times v_{t}^{2 *}-h_{2} \times v_{t}^{1 *}+\underline{c_{2}^{H}} \times n_{t},
\end{aligned}
$$

where $H$ represents the Hermitian of a matrix.

Following the received signal's correlation with $\underline{c_{1}}$ and $\underline{c_{2}}$, we arrive at two data symbols that are differentially decoded and then passed through the SP-symbol-to-bits demapper of Figure 1. Differential decoding is carried out by using the received data of two consecutive time slots as follows:

$$
\begin{aligned}
d_{t}^{1} & \times d_{t-1}^{1 *}+d_{t}^{2 *} \times d_{t-1}^{2} \\
& =\left(\left|h_{1}\right|^{2}+\left|h_{2}\right|^{2}\right) \times \sqrt{\left|v_{t-1}^{1}\right|^{2}+\left|v_{t-1}^{2}\right|^{2}} \times x_{1}+N_{1} \\
d_{t}^{1} & \times d_{t-1}^{2 *}-d_{t}^{2 *} \times d_{t-1}^{1} \\
& =\left(\left|h_{1}\right|^{2}+\left|h_{2}\right|^{2}\right) \times \sqrt{\left|v_{t-1}^{1}\right|^{2}+\left|v_{t-1}^{2}\right|^{2}} \times x_{2}+N_{2}
\end{aligned}
$$

where $N_{1}$ and $N_{2}$ are zero-mean complex-valued Gaussian random variables having variances of $\sigma_{N}^{2}=\sigma_{N_{1}}^{2}=\sigma_{N_{1}}^{2} \approx 2 \cdot h \cdot \sigma_{n}^{2}$, and we have $h=\left(\left|h_{1}\right|^{2}+\left|h_{2}\right|^{2}\right) \times \sqrt{\left|v_{t-1}^{1}\right|^{2}+\left|v_{t-1}^{2}\right|^{2}}$.

We can observe from Equations (13) and (14) that the proposed method guarantees achieving a diversity gain, since the two transmit antennas' signals are independently faded according to the values of $h_{1}$ and $h_{2}$, while using a low-complexity decoding algorithm. Since $c_{1}$ and $c_{2}$ are derived by appropriately concatenating the user-specific code $\underline{\mathrm{c}}$, no extra spreading codes are required for carrying out the STS operation and the two symbols of the two transmitters are transmitted in the same time slot.

The above analysis has been carried out for the case of a single receive antenna, but it can be readily extended for an arbitrary number of receive antennas, where the resultant signals are appropriately combined, before passing them to the decoder.

\section{ITERATIVE DEMAPPING}

Again, for the sake of simplicity, a system having a single receive antenna is considered, although its extension to several receive antennas is feasible. As already discussed in the previous section, the DSTS detected signals can be represented by Equations (13) and (14).

A received sphere-packed symbol $r$ is then constructed from the estimates $\tilde{x}_{1}$ and $\tilde{x}_{2}$ using Equation (2) as

$$
r=T^{-1}\left(\tilde{x}_{1}, \tilde{x}_{2}\right),
$$

where $r=\left\{\left[\tilde{a}_{1}, \tilde{a_{2}}, \tilde{a_{3}}, \tilde{a_{4}}\right] \in R^{4}\right\}$. The received sphere-packed symbol $r$ can be written as

$$
r=h \cdot \sqrt{\frac{2 L}{E}} \cdot s^{l}+N
$$

where we have $h=\left(\left|h_{1}\right|^{2}+\left|h_{2}\right|^{2}\right) \times \sqrt{\left|v_{t-1}^{1}\right|^{2}+\left|v_{t-1}^{2}\right|^{2}}, s^{l} \in S$, $0 \leq l \leq L-1$, and $N$ is a four-dimensional Gaussian random variable having a variance of $\sigma_{N}^{2} \approx 2 \cdot h \cdot \sigma_{n}^{2}$, since the SP symbol constellation $S$ is four-dimensional.

According to Equation (16), the conditional PDF $P\left(r \mid s^{l}\right)$ of $r$ is given by

$$
\begin{aligned}
p\left(r / s^{l}\right) & =\frac{1}{\left(2 \pi \sigma_{N}^{2}\right)^{\frac{N_{D}}{2}}} e^{-\frac{1}{2 \sigma_{N}^{2}}\left(r-\alpha \cdot s^{l}\right)^{2}} \\
& =\frac{1}{\left(2 \pi \sigma_{N}^{2}\right)^{\frac{N_{D}}{2}}} e^{-\frac{1}{2 \sigma_{N}^{2}}\left(\sum_{i=1}^{4}\left(\tilde{a}_{i}-\alpha \cdot a_{i}\right)^{2}\right)}
\end{aligned}
$$

where we have $\alpha=h \cdot \sqrt{\frac{2 L}{E}}$ and $N_{D}=4$, since a four-dimensional symbol constellation is used.

The SP symbol $r$ carries $B$ channel-coded bits $\mathbf{b}=b_{0, \ldots, B-1} \in$ $\{0,1\}$. The LLR-value of bit $k$ for $k=0, \ldots, B-1$ can be written as [10]

$$
L\left(b_{k} / r\right)=L_{a}\left(b_{k}\right)+\ln \frac{\sum_{s^{l} \in S_{1}^{k}} p\left(r / s^{l}\right) \cdot e^{\sum_{j=0, j \neq k}^{B-1} b_{j} L_{a}\left(b_{j}\right)}}{\sum_{s^{l} \in S_{0}^{k}} p\left(r / s^{l}\right) \cdot e^{\sum_{j=0, j \neq k}^{B-1} b_{j} L_{a}\left(b_{j}\right)}},
$$




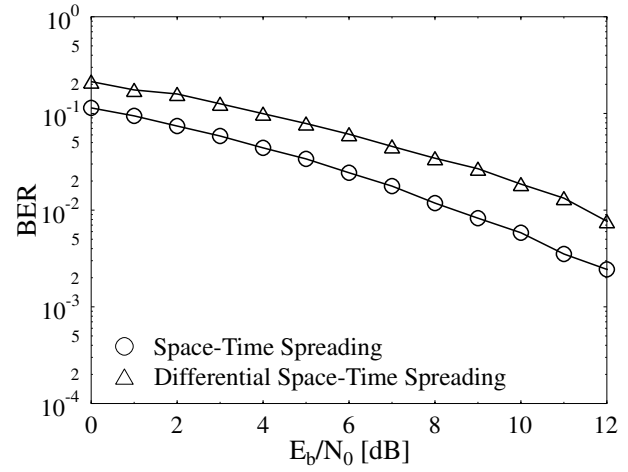

Figure 3: Performance Comparison of the Coherent and the Differential STS Schemes Using the System Parameters Outlined in Table 1.

\begin{tabular}{|l|r|}
\hline Modulation & Sphere Packing with $L=16$ \\
\hline No. of Transmitters & 2 \\
\hline No. of Receivers & 1 \\
\hline Channel & Correlated Rayleigh Fading \\
\hline $\begin{array}{l}\text { Normalised Doppler } \\
\text { frequency }\end{array}$ & 0.01 \\
\hline $\begin{array}{l}\text { Outer channel Code } \\
\text { Generator }\end{array}$ & RSC, $(2,1,5)$ \\
\hline Spreading Code & $\left(G_{r}, G\right)=(35,23)_{8}$ \\
\hline Spreading Factor & Walsh Code \\
\hline Number of users & 8 \\
\hline System throughput & 4 \\
\hline
\end{tabular}

Table 1: System parameters

where $S_{1}^{k}$ and $S_{0}^{k}$ are subsets of the symbol constellation $S$ such that $S_{1}^{k} \triangleq\left\{s^{l} \in S: b_{k}=1\right\}$ and likewise, $S_{0}^{k} \triangleq\left\{s^{l} \in S: b_{k}=\right.$ $0\}$. In other words, $S_{i}^{k}$ represents all symbols of the set $S$, where we have $b_{k} \in\{0,1\}, k=0, \ldots, B-1$. Using Equation (17), we can write Equation (18) as

$$
\begin{aligned}
& L\left(b_{k} / r\right) \\
= & L_{a}\left(b_{k}\right) \\
+ & \ln \frac{\sum_{s^{l} \in S_{1}^{k}} \exp \left[-\frac{1}{2 \sigma_{N}^{2}}\left(r-\alpha \cdot s^{l}\right)^{2}+\sum_{j=0, j \neq k}^{B-1} b_{j} L_{a}\left(b_{j}\right)\right]}{\sum_{s^{l} \in S_{0}^{k}} \exp \left[-\frac{1}{2 \sigma_{N}^{2}}\left(r-\alpha \cdot s^{l}\right)^{2}+\sum_{j=0, j \neq k}^{B-1} b_{j} L_{a}\left(b_{j}\right)\right]} \\
= & L_{M, a}+L_{M, e} .
\end{aligned}
$$

Finally, the max-log approximation of Equation (19) is as follows

$$
\begin{aligned}
& L\left(b_{k} / r\right) \\
= & L_{a}\left(b_{k}\right) \\
+ & \max _{\sum_{s^{l} \in S_{1}^{k}}}\left[-\frac{1}{2 \sigma_{N}^{2}}\left(r-\alpha \cdot s^{l}\right)^{2}+\sum_{j=0, j \neq k}^{B-1} b_{j} L_{a}\left(b_{j}\right)\right] \\
- & \max _{\sum_{s^{l} \in S_{0}^{k}}}\left[-\frac{1}{2 \sigma_{N}^{2}}\left(r-\alpha \cdot s^{l}\right)^{2}+\sum_{j=0, j \neq k}^{B-1} b_{j} L_{a}\left(b_{j}\right)\right] .
\end{aligned}
$$

\section{RESULTS AND DISCUSSION}

Without loss of generality, we considered an SP modulation scheme associated with $L=16$ and using two transmit as well as a single receiver antenna in order to demonstrate the performance improvements achieved by the proposed system. All simulation parameters are listed in Table 1. Since the space-time signal of Equa-

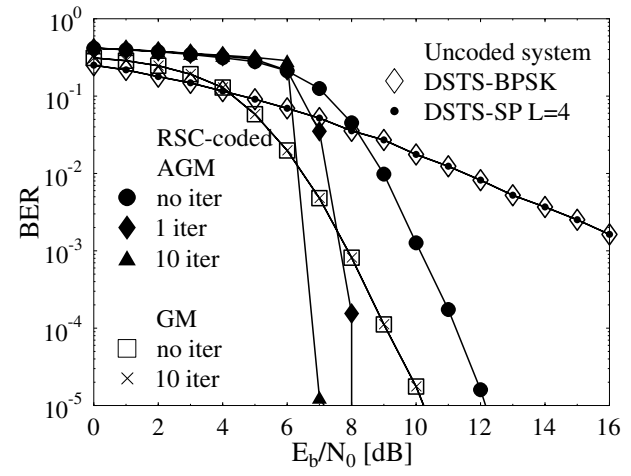

Figure 4: Performance comparison of various Gray Mapping (GM) and Anti-Gray Mapping (AGM) based convolutional-coded DSTS-SP schemes in conjuction with $L=16$ against an identical-throughput 2BPS uncoded DSTS-SP scheme using $L=4$, while using an interleaver length of D $=100000$ bits for a variable number of iterations and using the system parameters outlined in Table 1.

tion (3) is multiplied by a factor that is inversely proportional to $\sqrt{E}$, namely by $\sqrt{\frac{2 L}{E}}$, it is desirable to choose a specific subset of $L=16$ points from the entire set of legitimate constellation points hosted by $D_{4}$, which results in the minimum total energy. It was shown in [13] that there is a total of 24 legitimate symbols ${ }^{2}$ hosted by $D_{4}$ having an identical minimum energy of $E=2$. We used a computer search for determining the optimum choice of the $L=16$ points out of the possible 24 points, which possess the highest minimum Euclidean distance, hence minimising the error probability.

Figure 3 provides a performance comparison between the coherent STS and the proposed DSTS scheme. The results are presented for the SP constellation of $L=16$ and using the system parameters of Table 1 . The $3 d B$ SNR loss imposed by the non-coherent scheme clearly observed in the figure can be physically justified by considering the noise factors $N_{1}$ and $N_{2}$, in Equations (13) and (14), that have twice the noise power given by $\sigma_{N}^{2} \approx 2 \cdot h \cdot \sigma_{n}^{2}$ compared to that of the corresponding coherent detector. However, this $3 d B$ SNR loss may be considered as a worthwhile trade off for the sake of eliminating the channel estimation complexity.

Figures 4 and 5 compare the attainable performance of the proposed convolutional-coded DSTS-SP scheme employing both Anti-Gray Mapping (AGM) and Gray Mapping (GM) of the bits to the SP symbol, which are also contrasted to that of an identicalthroughput 2 Bit Per Symbol (2BPS) uncoded DSTS-SP scheme using $L=4$ and a conventional DSTS-BPSK design transmitting two independent BPSK symbols over the two antennas, when communicating over a correlated Rayleigh fading channel. In Figure 4 , an interleaver depth of $D=10^{6}$ bits was employed and a normalised Doppler frequency of $f_{D}=0.01$ was used. Observe in the figure that the two Gray Mapping (GM) based DSTSSP BER curves are exactly the same, when $I=0$ as well as $I=10$ turbo-detection iterations were employed. By contrast, Anti-Gray Mapping achieved a substantial performance improvement in conjunction with iterative demapping and decoding. Explicitly, Figure 4 demonstrates that a coding advantage of about $17.8 d B$ was achieved at a BER of $10^{-5}$ after $I=10$ iterations by the convolutional-coded AGM DSTS-SP system over both the uncoded DSTS-SP and the DSTS-BPSK schemes. Additionally,

\footnotetext{
${ }^{2}$ In simple terms, the sphere centred at $(0,0,0,0)$ has 24 spheres around it, centred at the points $(+/-1,+/-1,0,0)$, where any choice of signs and any ordering of the coordinates is legitimate [7, p.9].
} 


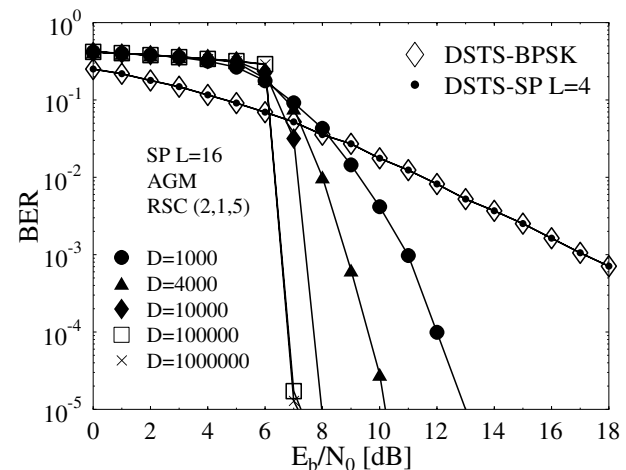

Figure 5: Performance comparison of the Anti-Gray Mapping (AGM) based convolutional-coded DSTS-SP scheme in conjuction with $L=16$ against an identical-throughput 2BPS uncoded DSTS-SP scheme using $L=4$, for a variable interleaver length $\mathrm{D}$ invoking $I=10$ iterations and using the system parameters outlined in Table 1.

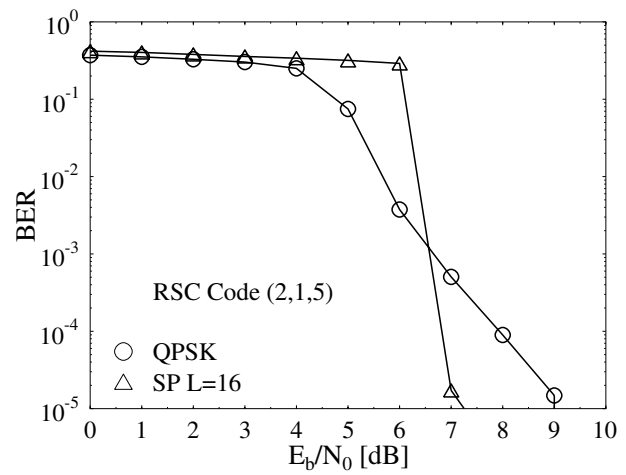

Figure 6: Performance comparison of Anti-Gray Mapping (AGM) based convolutional-coded DSTS scheme in conjuction with SP $L=16$ against an identical-throughput 2BPS DSTS-QPSK scheme, while using an interleaver length of $\mathrm{D}=100000$ bits for $I=10$ iterations and using the system parameters outlined in Table 1.

a coding advantage of approximately $3 d B$ was attained over the 2BPS-throughput convolutional-coded GM DSTS-SP scheme. Figure 5 provides a performance comparison of the proposed convolutional-coded DSTS-SP scheme employing both Anti-Gray Mapping (AGM) based on the beneficial effect of the long interleaver together with an identical-throughput 2 Bit Per Symbol (2BPS) uncoded DSTS-SP scheme and a conventional DSTS-BPSK scheme. The figure demonstrates that there is no significant coding gain beyond the interleaver length of $10^{5}$ bits.

Finally, as suggested in the introduction, the proposed turbodetected DSTS-SP scheme provides an improved performance over an equivalent DSTS scheme dispensing with SP modulation, as evidenced in Figure 6, demonstrating that the DSTS-SP scheme using $L=16$ exhibits an $E_{b} / N_{o}$ gain of around $1.9 d B$ at a $B E R$ of $10^{-5}$ over the identical-throughput 2BPS DSTS-QPSK scheme.

\section{CONCLUSION}

In this paper we proposed a novel system that exploits the advantages of both iterative demapping and turbo detection [10], as well as those of the DSTS-SP scheme developed. The proposed DSTS scheme benefits from a substantial diversity gain, while using two transmit antennas without the need for any CSI. The DSTS suffers from an approximately $3 d B$ performance loss as compared to coherent detection. However, this may be considered as a worthwhile trade-off for the sake of eliminating the high-complexity channel estimation. Moreover, our investigations demonstrated that signifi- cant performance improvements may be achieved, when the AGM DSTS-SP scheme is combined with outer channel decoding and iterative SP-symbol-to-bit demapping, as compared to the GrayMapping based systems. When using an appropriate bit-to-SPsymbol mapping scheme and $I=10$ turbo detection iterations, an $E_{b} / N_{0}$ gain of about $17.8 \mathrm{~dB}$ was obtained by the convolutionalcoded STBC-SP scheme over the identical-throughput 2 bit/symbol uncoded STBC-SP benchmarker scheme.

Our future research will consider the design of similar QAM schemes for transmission over dispersive channels as well as blind channel estimation for coherent STS schemes.

\section{REFERENCES}

[1] L. Hanzo, T. H. Liew, and B. L. Yeap, Turbo Coding, Turbo Equalisation and Space-Time Coding: for Transmission over Fading Channels. Chichester, England: John Wiley and Sons Ltd and IEEE Press, NY, USA, 2002

[2] S. Alamouti, "A simple transmit diversity technique for wireless communications," IEEE Journal on Selected Areas in Communications, vol. 16, no. 8, pp. 1451-1458, 1998.

[3] V. Tarokh, H. Jafarkhani, and A. Calderbank, "Space-time block codes from orthogonal designs," IEEE Transactions on Information Theory, vol. 45, pp. 1456-1467, Jul 1999.

[4] V. Tarokh, H. Jafarkhani, and A. Calderbank, "Space-time block coding for wireless communications: performance results," IEEE Journal on Selected Areas in Communications, vol. 17, pp. 451-460, Mar 1999.

[5] B. M. Hochwald, G. Gaire, B. Hassibi, and E. T. L. Marzetta, "Special issue on space-time transmission, reception, coding and signal processing," IEEE Transactions on Information Theory, pp. 23292806 , Oct 2003

[6] V. Tarokh and H. Jafarkhani, "A differential detection scheme for transmit diversity," in IEEE Journal on Selected Areas in Communications, vol. 18, pp. 1169-1174, July 2000.

[7] B. Hochwald, T. Marzetta, and C. Papadias, "A transmitter diversity scheme for wideband CDMA systems based on space-time spreading," IEEE Journal on Selected Areas in Communications, vol. 19, pp. 48-60, Jan 2001.

[8] W. Su, Z. Safar, and K. J. R. Liu, "Space-time signal design for timecorrelated Rayleigh fading channels," in IEEE International Conference on Communications, vol. 5, (Anchorage, Alaska), pp. 31753179, 2003.

[9] S. L. Goff, A. Glavieux, and C. Berrou, "Turbo-codes and high spectral efficiency modulation," in Proceedings of International Conference on Communications, (New Orleans, United States), pp. 645649, May 1994.

[10] S. ten Brink, J. Speidel, and R.-H. Yan, "Iterative demappping and decoding for multilevel modulation," in IEEE Global Telecommunications Conference, vol. 1, (Sydney, Australia), pp. 579-584, 8-12 Nov 1998.

[11] O. Alamri, B. L. Yeap, and L. Hanzo, "Turbo detection of channelcoded space-time signals using sphere packing modulation," in IEEE Vehicular Technology Conference, vol. 4, (Los Angeles, USA), pp. 2498-2502, Sep 2004.

[12] P. Robertson, E. Villebrun, and P. Hoeher, "A comparison of optimal and sub-optimal MAP decoding algorithms operating in the log domain," in Proceedings of International Conference on Communications, (Seattle, USA), pp. 1009-1013, Jun 1995.

[13] J. H. Conway and N. J. Sloane, Sphere Packings, Lattices and Groups. Springer-Verlag, 1999.

[14] C.-S. Hwang, S. H. Nam, J. Chung, and V. Tarokh, "Differential space time block codes using nonconstant modulus constellations," in IEEE Transactions on Signal Processing, vol. 51, pp. 2955-2964, Nov 2003.

[15] L. Hanzo, S. X. Ng, T. Keller, and W. Webb, Qaudrature Amplitude Modulation: From Basic to Adaptive Trellis-Coded, Turbo Equalised and Space-Time Coded OFDM, CDMA and MC-CDMA Systems, 2nd Edition. Chichester, England: John Wiley and Sons Ltd and IEEE Press, 2004 